\title{
Generalizing the concept of quantum triads
}

\author{
Radek Šlesinger \\ Masaryk University \\ Brno, Czech Republic \\ xslesing@math.muni.cz
}

\begin{abstract}
The concept of quantum triad has been introduced by D. Kruml [6], where for a given pair of quantal $\S^{1}$ modules $L, R$ over a common quantale $Q$, endowed with a bimorphism (a 'bilinear map') to $Q$, a construction equipping $L$ and $R$ with additional module structure and another bimorphism, both compatible with the existing bimorphism and action of the quantale, was presented. As the original concept was only defined in a specific setting of categories of quantale modules, we extend it to a more universal one, which can be applied to other common algebraic structures.

In what follows, we assume that all the categories are concrete (via the forgetful functor $|-|$ into Set $)$. Let $\mathbf{V}=\left(\mathbf{V}, \otimes_{\mathcal{V}}, I_{\mathbf{V}}\right)$ be a closed symmetric monoidal category and $\mathbf{C}$ be a subcategory of $\mathbf{V}$, enriched in $\mathbf{V}$. Suppose $M$ is a monoid in $\mathbf{V}$ (viewed as a $\mathbf{V}$-category with a single object $\star_{M}$ ). We call an object $A$ of $\mathbf{C}$ a left (right) module over $M$ if there is a $\mathbf{V}$-enriched functor from $M\left(M^{o p}\right)$ to $\mathbf{C}$ that maps $\star_{M}$ to $A$. Next, $A$ is a $M, N$-bimodule if there is a $\mathbf{V}$-enriched bifunctor from $M \times N^{o p}$ to $\mathbf{C}$ mapping $\left(\star_{M}, \star_{N}\right)$ to $A$. A map (in Set) $f:|A| \times|B| \rightarrow|C|$ is a C-bimorphism[1] if every map $f(a,-):|B| \rightarrow|C|$ and $f(-, b):|A| \rightarrow|C|$ arises from a C-morphism $f_{A}: B \rightarrow C$ or $f_{B}: A \rightarrow C$, respectively, as $\left|f_{A}\right|$ or $\left|f_{B}\right|$.
\end{abstract}

Definition. Let $\mathbf{V}, \mathbf{C}$ be as above, $L, R$ be a left and a right module over a $\mathbf{V}$-monoid $T$. Further, let $\tau$ be a $\mathbf{C}$-bimorphism from $L \times R$ to $T$. Then the tuple $(L, R, \tau, T)$ is called a triad. If there exists a monoid $S$ in $\mathbf{V}$ together with a $\mathbf{V}$-bimorphism $\sigma: R \times L \rightarrow S$ which makes $L$ a T, S-bimodule and $R$ a $S, T$-bimodule, and is compatible with $\tau$ (this means, for instance 'RLR': for any $l \in L$ and $r, r^{\prime} \in R, \sigma(r, l) \cdot r^{\prime}=r \cdot \tau\left(l, r^{\prime}\right)$, and a few similar conditions), we call $(S, \sigma)$ a solution of the triad.

Existence of solutions together with additional properties can be proved when certain assumptions on the category $\mathbf{C}$ are satisfied:

Proposition. 1. Let $\mathbf{C}$ have tensor products over $T$, i.e., it has coequalizers of morphisms $R \otimes T \otimes L \rightrightarrows R \otimes L$ obtained from $T$ acting on $R$ and $L$, respectively. Then the universal property of the tensor product provides a solution $\left(S_{0}, \sigma_{0}\right)$ given by $R \otimes_{T} L$ and $\sigma_{0}:(r, l) \mapsto$ $r \otimes_{T} l$, which is initial - for any solution $(S, \sigma)$ there is a morphism $s_{0}: S_{0} \rightarrow S$ such that $\sigma=s_{0} \circ \sigma_{0}$. In this case, the solution belongs to $\mathbf{C}$. Multiplication in $S_{0}$ and action of $S_{0}$ on $R$ are as follows:

- $\left(r_{1} \otimes_{T} l_{1}\right) \cdot\left(r_{2} \otimes_{T} l_{2}\right)=r_{1} \cdot \tau\left(l_{1}, r_{2}\right) \otimes_{T} l_{2}=r_{1} \otimes_{T} \tau\left(l_{1} r_{2}\right) \cdot l_{2}$,

- $\left(r \otimes_{T} l\right) \cdot r^{\prime}=r \cdot \tau\left(l, r^{\prime}\right)$ and $l^{\prime} \cdot\left(r \otimes_{T} l\right)=\tau\left(l, r^{\prime}\right) \cdot l$.

2. $S_{1}=\{(\alpha, \beta) \in \mathbf{C}(L, L) \times \mathbf{C}(R, R) \mid \tau(\alpha(l), r)=\tau(l, \beta(r))$ for any $l \in L, r \in R\}$ with $\sigma_{1}:(l, r) \mapsto((-l) r, l(r-))$ is another solution. It is terminal, since any monoid acting on $L(R)$ and satisfying the compatibility conditions can be represented in $S_{1}$. Multiplication and action of $S_{1}$ are following:

${ }^{1}$ A quantale is a complete join-semilattice equipped with associative binary multiplication distributing over arbitrary joins, or, a semigroup in the category of complete join-semilattices. For more information on quantales and their modules, see e. g. 7, 8, 9]. 
- $\left(\alpha_{1}, \beta_{1}\right) \cdot\left(\alpha_{2}, \beta_{2}\right)=\left(\alpha_{2} \circ \alpha_{1}, \beta_{1} \circ \beta_{2}\right)$,

- $(\alpha, \beta) r=\beta(r)$ and $l(\alpha, \beta)=\alpha(l)$.

It has been shown in [6] that $S_{0}$ and $S_{1}$ form a so-called couple of quantales (see also [4]) and that $S$ is a solution iff $S_{0} \rightarrow S_{1}$ factorizes through $S$.

An example application in the original paper was reconstructing a quantale from given sets of its right-, left- and two-sided elements, and the base category was SupLat, complete joinsemilattices with join-preserving maps. Here we extend this construction to other categories.

Example 1. Let $V$ be a linear space over a field $K$, equipped with an inner product $\langle-,-\rangle: V \times$ $V \rightarrow K$. The solutions of the triad $(V, V,\langle-,-\rangle, K)$ are then provided by linear operators on $V$, namely $S_{0}$ consisting of elements $u_{1} \otimes_{K} v_{1}+\cdots+u_{n} \otimes_{K} v_{n}$, each acting on $V$ as making a linear combination of $u_{1}, \ldots, u_{n}$ with coefficients $\left\langle v_{i}, w\right\rangle$, and $S_{1}=\{(\alpha, \beta) \in \operatorname{End}(V) \times \operatorname{End}(V) \mid$ $\langle\alpha(u), v\rangle=\langle u, \beta(v)\rangle\}$, the ring formed by pairs of adjoint linear operators on $V$ (with reversed order of composition on the first component).

In many situations, the categories $\mathbf{C}$ and $\mathbf{V}$ coincide, like in the original case of quantales (with SupLat, the category of complete join-semilattices), rings (with $\mathbf{A b}$, the category of abelian groups), or ordinary monoids (with Set), and the whole category of solutions is contained in $\mathbf{C}$.

Using more recent results on existence of tensor products for some algebras of quantum logic, other categories allowing formation of triads can be considered. For instance, we take the category effect algebra: ${ }^{2}$ with effect algebra morphisms, EffAlg. Here the distinction of $\mathbf{V}$ and $\mathbf{C}$ is required as EffAlg has tensor products (when trivial effect algebras with $0=1$ are allowed) and is monoidal with the 2-element algebra $\{0,1\}$ being the tensor unit, however, it is not closed 5. The enriching category $\mathbf{V}$ is now that of generalized effect algebras (GenEffAlg).

Example 2. A state ('probability measure') on an effect algebra $E$ is an effect-algebra morphism (preserving $\oplus$ if it is defined, ${ }^{\perp}, 0$ and 1) $s: E \rightarrow[0,1]$. A convex effect algebra is an effect algebra with action of the real interval $[0,1]$ (viewed as an effect algebra, with $a \oplus b$ defined if $a+b \leq 1$ ), which becomes a monoid in EffAlg under standard multiplication of reals. Having two convex effect algebras with states $\left(E, s_{E}\right)$ and $\left(F, s_{F}\right)$, one obtains an effect algebra with a state $\left(E \otimes F, s_{E} \cdot s_{F}\right)[2$. This forms a triad $E \times F \rightarrow[0,1]$. Like in the previous example, its special solutions are $S_{0}=F \otimes_{[0,1]} E$, and $S_{1}$ - pairs of endomorphisms acting on the components of the tensor product, mutually adjoint wrt. the state on $E \otimes F$.

\section{References}

[1] Bernhard Banaschewski and Evelyn Nelson. Tensor products and bimorphisms. Canadian Mathematical Bulletin, 19(4):385-402, 1976.

[2] Anatolij Dvurečenskij. Tensor product of difference posets and effect algebras. International Journal of Theoretical Physics, 34(8):1337-1348, 1995.

[3] Anatolij Dvurečenskij and Sylvia Pulmannová. New Trends in Quantum Structures. Mathematics and Its Applications. Kluwer Academic Publishers, Dordrecht, 2000.

\footnotetext{
${ }^{2}$ Briefly, a partial commutative monoid (pcmon) is an algebra $(M, \oplus, 0)$ where $0 \in M$ and $\oplus$ is a partially defined commutative and associative operation with 0 as a neutral element. A conical and cancellative pcmon is called a generalized effect algebra. A pcmon $E$ is called an effect algebra if it is equipped with a unary operation $\perp$ and an element 1 such that $x \oplus y=1$ iff $y=x^{\perp}$, and having $x \oplus 1$ defined implies $x=0$. A symmetric binary relation (orthogonality) can be defined on $E$ by $a \perp b$ iff $a \oplus b$ is defined. A frequent instance of effect algebras is the one of bounded positive operators on a Hilbert space. For reference on effect algebras and other quantum structures, we suggest [3].
} 
[4] Jeff M. Egger and David Kruml. Girard couples of quantales. Applied Categorical Structures, 18(2):123-133, 2010.

[5] Bart Jacobs and Jorik Mandemaker. Coreflections in algebraic quantum logic. Foundations of Physics, 42(7):932-958, 2012.

[6] David Kruml. Quantum triads: an algebraic approach. http://arxiv.org/abs/0801.0504.

[7] David Kruml and Jan Paseka. Algebraic and categorical aspects of quantales. In Handbook of Algebra, volume 5, pages 323-362. North-Holland, 2008.

[8] Kimmo I. Rosenthal. Quantales and their applications. Pitman Research Notes in Mathematics. Longman Scientific \& Technical, New York, 1990.

[9] Sergey A. Solovyov. On the category Q-Mod. Algebra universalis, 58:35-58, 2008. 